\title{
Carleman estimate for a one-dimensional system of $m$ coupled parabolic PDEs with $B V$ diffusion coefficients
}

\section{Hichem Ramoul}

\section{"Correspondence:}

ramoul2477@yahoo.fr

Département de Mathématiques et

Informatique, Faculté des Sciences

et Technologie, Université Abbes

Laghrour-Khenchela, Route de

Constantine, Khenchela, 40000

Algeria

Laboratoire de Mathématiques Appliquées, Université Badji

Mokhtar, BP 12, Annaba, 23000 Algeria

\begin{abstract}
This paper is devoted to deriving global Carleman estimate for a one-dimensional linear coupled parabolic system of $m$ equations with bounded variations (BV) diffusion coefficients. This kind of estimate is a generalization of the scalar result (Le Rousseau in J. Differ. Equ. 233:417-447, 2007). The key ingredient is to derive a global Carleman estimate for piecewise- $\mathcal{C}^{1}$ diffusion coefficients based on the construction of a suitable weight function. The Carleman estimate in the case of $B V$ diffusion coefficients is then obtained using the approach of $B V$ diffusion coefficients by piecewise-constant coefficients. This Carleman estimate is used to show the observability inequality which yields the controllability result.
\end{abstract}

MSC: 35K40; 26A45; 93B07

Keywords: Carleman estimate; parabolic equations; BV coefficients; observability; controllability

\section{Introduction and notations}

In this paper we deal with one-dimensional $m$ coupled parabolic equations with bounded variations $(B V)$ diffusion coefficients.

Let $\Omega=(0,1) \subset \mathbb{R}$ be a one-dimensional bounded domain, and we assume that $T>0$. Let us consider the following notations: $Q=\Omega \times(0, T), \Gamma=\{0,1\}$ and $\Sigma=\Gamma \times(0, T)$.

For $m \geq 1$ given, we denote by $\mathcal{A}_{j}=-\partial_{x}\left(k_{j} \partial_{x}\right)$ the elliptic operator formally defined on $L^{2}(\Omega), 1 \leq j \leq m$, with the domain of $\mathcal{A}_{j}$ given by

$$
D\left(\mathcal{A}_{j}\right)=\left\{v \in H_{0}^{1}(\Omega) ; k_{j} \partial_{x} v \in H^{1}(\Omega)\right\}, \quad j=1, \ldots, m .
$$

The diffusion coefficients $k_{j}=k_{j}(x)(j=1, \ldots, m)$ are assumed to be of $B V$ and satisfy the following.

\section{Assumption 1.1}

$$
0<k_{j, \min } \leq k_{j} \leq k_{j, \max }<\infty, \quad j=1, \ldots, m \text {. }
$$

(o 2014 Ramoul; licensee Springer. This is an Open Access article distributed under the terms of the Creative Commons Attribution License (http://creativecommons.org/licenses/by/2.0), which permits unrestricted use, distribution, and reproduction in any medium, provided the original work is properly credited. 
Let us introduce the following matrix operator $\mathcal{A}$ defined by

$$
\mathcal{A}=\left(\begin{array}{cccc}
\mathcal{A}_{1} & 0 & \cdots & 0 \\
0 & \mathcal{A}_{2} & & \vdots \\
\vdots & & \ddots & 0 \\
0 & \cdots & 0 & \mathcal{A}_{m}
\end{array}\right)
$$

The domain of $\mathcal{A}$ is given by $D(\mathcal{A}):=\prod_{j=1}^{m} D\left(\mathcal{A}_{j}\right)$.

We denote $H=\left(L^{2}(\Omega)\right)^{m}$ and let us consider the following linear parabolic system:

$$
\begin{cases}\partial_{t} y+\mathcal{A} y=L y+F & \text { in } Q \\ y(x, 0)=y_{0}(x) & \text { in } \Omega\end{cases}
$$

where $y(\cdot, t)=\left(y_{j}(\cdot, t)\right)_{1 \leq j \leq m} \in D(\mathcal{A})$ for $y_{0}=\left(y_{0, j}\right)_{1 \leq j \leq m} \in H, L=\left(a_{j k}(x, t)\right)_{1 \leq j, k \leq m} \in$ $\left(L^{\infty}(Q)\right)^{m^{2}}$ and $F=\left(f_{j}(x, t)\right)_{1 \leq j \leq m} \in\left(L^{2}(Q)\right)^{m}$ for all $t \in(0, T)$.

Let us observe that, under Assumption 1.1, for each $y_{0} \in H$ and $F \in\left(L^{2}(Q)\right)^{m}$, system (1.1) admits a unique weak solution $y \in L^{2}\left((0, T) ;\left(H_{0}^{1}(\Omega)\right)^{m}\right) \cap \mathcal{C}([0, T] ; H)$ (see, e.g., [1]).

The main goal of this paper is to prove a global Carleman estimate for the operator $\partial_{t}+\mathcal{A}$ with an interior observation region $\omega \times(0, T)$, where $\omega$ is a non-empty open subset of $\Omega$ and such that $k_{j}$ are of class $\mathcal{C}^{1}$ on $\bar{\omega}$.

The Carleman estimate for piecewise regular diffusion coefficients is established by Doubova et al. in [2]. In this work, the authors considered a scalar parabolic equation. They obtained observability inequality and controllability results by adding assumption on the monotonicity of the coefficient (i.e., the observability is supported in the region where the diffusion coefficient is the lowest). To obtain these results, the authors introduced a non-smooth weight function $\beta$, assuming that it satisfies the same transmission condition as the solution of a parabolic equation. An inverse problem for such a parabolic equation was studied in [3]. In the same direction, we can also cite the work [4] of Bellassoued and Yamamoto which is devoted to determining a source term using the Carleman estimate established in [2]. In 2007, a new Carleman estimate was established by Benabdallah et al. [5] for the one-dimensional heat equation with a discontinuous diffusion coefficient. In this work the authors relaxed the monotonicity assumption on the diffusion coefficient by constructing a specific non-smooth weight function $\beta$. This function $\beta$ satisfies suitable trace properties depending on the jumps of the derivatives of $\beta$ at the singular points of the diffusion coefficient. In higher dimensions $(n \geq 2)$, Le Rousseau and Robbiano in [6] showed that the monotonicity assumption on the diffusion coefficients can be relaxed and the observation region can be chosen independently of the jump's sign of the diffusion coefficient. In the same way, we cite the work [7] about Carleman estimates in stratified media. In [8], Le Rousseau generalized the results obtained in [5] for the case of bounded variations diffusion coefficient $(B V)$. In Le Rousseau's paper, the author constructed a limit weight function as he approached $B V$ coefficient by piecewise-constant coefficient. However, the relaxation of the monotonicity condition in the case of bounded variations diffusion coefficient in any dimension $n \geq 2$ remains open.

For the first time, a Carleman-type estimate with one observation in parabolic systems was introduced by Ammar Khodja et al. $[9,10]$ where the authors used this estimate to 
establish observability inequality and deduce a controllability result by one control force. We also refer to [11,12] for this kind of works. In paper [13], Cristofol et al. obtained a new Carleman-type estimate with one observation acting on a subdomain $\omega$ of $\mathbb{R}^{n}(n \leq 3)$ for a $2 \times 2$ reaction-diffusion system. They used this estimate for simultaneous identification of one parameter and initial conditions. We also cite the article [14], which represents an improvement of the work [13]. It is about determining two coefficients by observation data of only one component in a nonlinear $2 \times 2$ parabolic system. In the same direction, we can cite the works $[15,16]$.

If the observation region $\omega$ is replaced by $\gamma \subset \Gamma$, the Carleman estimate with $(m-1)$ observations for a system of $m(m \geq 2)$ coupled parabolic equations remains an open question.

In the same way, we cite the recent work [17] about an inverse problem for a onedimensional coupled parabolic system (two equations) with discontinuous conductivities (assumed to be $L^{\infty}$ ). The paper [17] is devoted to proving the stability result using the Carleman estimate (with the observation of only one component) based on an adequate choice of weight function which is the same for each equation of a parabolic system. However, the authors needed additional assumptions on this Carleman weight function, and the method that was developed is completely different with respect to the approach obtained in our paper.

Roughly speaking, the aim of our paper is to extend the results obtained in [8] to the case of $m$ coupled parabolic equations. One of the main difficulties in extending the scalar result comes from the fact that the weight function $\beta$ has to be chosen the same for each equation and depends on the jumps of diffusion coefficients. Moreover, since the jump discontinuities may be located at different points for the diffusion coefficients $k_{j}(1 \leq j \leq$ $m$ ), this created an additional difficulty to find our weight function.

The major novelty of our work is to prove a global Carleman estimate (with $m$ observations) in the case of $B V$ diffusion coefficients $k_{j}(1 \leq j \leq m)$ for the operator $\partial_{t}+\mathcal{A}$. In a first step, we derive a global Carleman estimate (with $m$ observations) in the case of piecewise$\mathcal{C}^{1}$ diffusion coefficient. The main result, in this case, is Lemma 2.1, where we prove the existence of a suitable weight function for $m$ coupled parabolic equations in the case of piecewise- $\mathcal{C}^{1}$ coefficients. By comparison with [8], the idea in the proof of Lemma 2.1 lies in the fact that we have used adapted choices (more general) (see formulas (2.7) and (2.8)) for checking the trace property (2.3) in the case of $m$ coupled parabolic equations. These choices are used later for constructing a function $\beta$ (see formulas (3.2) and (3.3)) in the case of $B V$ diffusion coefficients. The property (2.3) is needed to relax the condition of the monotonicity of the diffusion coefficients. In a second step, we follow the method developed in [8]. Formulas (3.2) and (3.3) yield an explicit expression of an approached weight function $\beta_{\varepsilon}$ that converges to a weight function $\beta$ (see Lemma 3.2). The function $\beta_{\varepsilon}$ allows us to establish a Carleman-type estimate (with $m$ observations) associated to the operator $\partial_{t}-\partial_{x}\left(k_{j, \varepsilon} \partial_{x}\right)$ with $k_{j, \varepsilon}(1 \leq j \leq m)$ piecewise constants that converge to the $B V$ diffusion coefficients $k_{j}$ in $L^{\infty}$-norm. At the end, we pass to the limit for each term in the Carleman estimate that holds for the operator $\partial_{t}-\partial_{x}\left(k_{j, \varepsilon} \partial_{x}\right)$ as $\left\|k_{j, \varepsilon}-k_{j}\right\|_{L^{\infty}}$ goes to zero. We then obtain the Carleman estimate for the operator $\partial_{t}+\mathcal{A}$ with a relaxation of the monotonicity of $B V$ diffusion coefficients $k_{j}$.

To our knowledge, the weight Carleman function and its proof in our work has not been proposed in the literature review. 
The article is organized as follows. In Section 2, we derive a Carleman estimate with $m$ observations in the case of piecewise- $\mathcal{C}^{1}$ diffusion coefficients. In Section 3, we prove a Carleman estimate with $m$ observations in the case of $B V$ diffusion coefficients. Finally, Section 4 is devoted to giving important comments and applications of our results on controllability for some parabolic systems.

\section{Global Carleman estimate with ' $m$ observations' in the case of piecewise- $\mathcal{C}^{1}$ diffusion coefficients}

In this section, we generalize the Carleman estimate obtained in [5] to a parabolic system. We prove here a global Carleman estimate in the case of piecewise- $\mathcal{C}^{1}$ diffusion coefficients for a system of $m$ coupled parabolic equations with an interior observation region $\omega \times$ $(0, T)$, where $\omega$ is a non-empty open subset of $\Omega$. In order to establish this estimate, we use similar arguments to those in [8] and [5] for constructing a suitable weight function in a subdomain of $\mathbb{R}$, which allows us to relax the monotonicity on the diffusion coefficients. The results obtained in this section are then used in the next section (the case of $B V$ diffusion coefficients).

Let $i=1, \ldots, n-1$ and $j=1, \ldots, m$. Let $a_{1}, \ldots, a_{n-1}$ with $0=a_{0}<a_{1}<a_{2}<\cdots<a_{n-1}<$ $a_{n}=1$.

We note : $\Omega_{i}=\left[a_{i}, a_{i+1}\right], S=\left\{a_{1}, \ldots, a_{n-1}\right\}, \Omega^{\prime}=\Omega \backslash S, Q^{\prime}=\Omega^{\prime} \times(0, T)$, and $S_{T}=S \times[0, T]$.

Let us consider system (1.1) formulated with the transmission conditions (TC) on $S_{T}$ (given by the fact that $y(\cdot, t)=\left(y_{j}(\cdot, t)\right)_{1 \leq j \leq m} \in D(\mathcal{A})$ ):

$$
\begin{cases}y_{j}\left(a_{i}^{-}\right)=y_{j}\left(a_{i}^{+}\right), & i=1, \ldots, n-1, j=1, \ldots, m, \\ k_{j}\left(a_{i}^{-}\right) \partial_{x} y_{j}\left(a_{i}^{-}\right)=k_{j}\left(a_{i}^{+}\right) \partial_{x} y_{j}\left(a_{i}^{+}\right), & i=1, \ldots, n-1, j=1, \ldots, m .\end{cases}
$$

The diffusion coefficients $k_{j}(j=1, \ldots, m)$ are assumed here to be piecewise- $\mathcal{C}^{1}$ such that $k_{j} \mid \Omega_{i} \in \mathcal{C}^{1}\left(\Omega_{i}\right)(i=0, \ldots, n-1)$ and satisfy Assumption 1.1.

Let us introduce the following set $\Lambda=\Lambda_{c} \cup \Lambda_{d}$, where

$$
\begin{aligned}
& \Lambda_{c}=\left\{\left(a_{i}, k_{j}\right) ; a_{i} \text { is a point of continuity of } k_{j}\right\}, \\
& \Lambda_{d}=\left\{\left(a_{i}, k_{j}\right) ; a_{i} \text { is a point of discontinuity of } k_{j}\right\} .
\end{aligned}
$$

Remark 2.1 If $\left(a_{i}, k_{j}\right) \in \Lambda_{c}$, the transmission conditions (TC) are then automatically satisfied.

We shall now prove the main result of this section. It concerns the construction of a suitable weight function.

Lemma 2.1 Let fixed $p \in\{0, \ldots, n-1\}$ such that $\left(a_{p}, a_{p+1}\right) \Subset \Omega$. Let $\omega_{0} \Subset \omega \Subset\left(a_{p}, a_{p+1}\right)$ be a non-empty open set. Then there exists a function $\widetilde{\beta} \in \mathcal{C}(\bar{\Omega})$ such that

$$
\begin{aligned}
& \left.\widetilde{\beta}\right|_{\Omega_{i}} \in \mathcal{C}^{2}\left(\Omega_{i}\right), \quad i=0, \ldots, n-1, \\
& \widetilde{\beta}>0 \quad \text { in } \Omega, \quad \widetilde{\beta}=0 \quad \text { on } \Gamma, \\
& \left.\widetilde{\beta}^{\prime}\right|_{\left[a_{p}, a_{p+1}\right]} \neq 0 \quad \text { in }\left[a_{p}, a_{p+1}\right] \backslash \omega_{0},
\end{aligned}
$$


$\left.\widetilde{\beta}^{\prime}\right|_{\Omega_{i}} \neq 0, \quad i \in\{0, \ldots, n-1\}, i \neq p$,

$\partial_{x} \widetilde{\beta}>0 \quad$ on the left-hand side of $\omega_{o}, \quad \partial_{x} \widetilde{\beta}<0 \quad$ on the right-hand side of $\omega_{o}$,

and the function $\widetilde{\beta}$ satisfies the following trace properties, $\forall l \in\{1, \ldots, m\}$ and some $\alpha>0$,

$$
\forall\left(a_{i}, k_{l}\right) \in \Lambda, \quad\left(A_{i}\left(k_{l}\right) u, u\right) \geq \alpha\|u\|^{2}, \quad i=1, \ldots, n-1,
$$

with $u=\left(u_{1}, u_{2}\right)^{t}, u_{1}, u_{2} \in \mathbb{R}$ and the matrices $A_{i}\left(k_{l}\right)$ are defined by

$$
A_{i}\left(k_{l}\right)=\left(\begin{array}{cc}
{\left[\widetilde{\beta}^{\prime}\right]_{a_{i}}} & \widetilde{\beta}^{\prime}\left(a_{i}^{+}\right)\left[k_{l} \widetilde{\beta}^{\prime}\right]_{a_{i}} \\
\widetilde{\beta}^{\prime}\left(a_{i}^{+}\right)\left[k_{l} \widetilde{\beta}^{\prime}\right]_{a_{i}} & \widetilde{\beta}^{\prime}\left(a_{i}^{+}\right)\left[k_{l} \widetilde{\beta}^{\prime}\right]_{a_{i}}^{2}+\left[k_{l}^{2}\left(\widetilde{\beta}^{\prime}\right)^{3}\right]_{a_{i}}
\end{array}\right), \quad i=1, \ldots, n-1,
$$

where $[\rho]_{a_{i}}=\rho\left(a_{i}^{+}\right)-\rho\left(a_{i}^{-}\right)$and $\widetilde{\beta^{\prime}}=\partial_{x} \widetilde{\beta}$.

Proof In the case of one equation ( $m=1$ ), the proof of the existence of such a function $\widetilde{\beta}$ is established in [8] and [5]. However, in our case ( $m$ coupled equations), the main difficulty is to find $\beta$ such that the trace property (2.3) is satisfied for all $l \in\{1, \ldots, m\}$.

Observe that the symmetric matrices $A_{i}\left(k_{l}\right)$ are positive definite if and only if

$$
\left[\widetilde{\beta}^{\prime}\right]_{a_{i}}>0 \quad \text { and } \quad \operatorname{det}\left(A_{i}\left(k_{l}\right)\right)>0 \quad \forall l \in\{1, \ldots, m\}
$$

Let us consider the following notations:

$$
X_{i}=\frac{\widetilde{\beta}^{\prime}\left(a_{i}^{-}\right)}{\widetilde{\beta}^{\prime}\left(a_{i}^{+}\right)}, \quad Y_{i, l}=\frac{k_{l}\left(a_{i}^{+}\right)}{k_{l}\left(a_{i}^{-}\right)}, \quad i=1, \ldots, n-1, l=1, \ldots, m,
$$

this leads to

$$
A_{i}\left(k_{l}\right)=\left(\begin{array}{cc}
\widetilde{\beta}^{\prime}\left(a_{i}^{+}\right)\left(1-X_{i}\right) & k_{l}\left(a_{i}^{-}\right)\left(\widetilde{\beta}^{\prime}\left(a_{i}^{+}\right)\right)^{2}\left(Y_{i, l}-X_{i}\right) \\
k_{l}\left(a_{i}^{-}\right)\left(\widetilde{\beta}^{\prime}\left(a_{i}^{+}\right)\right)^{2}\left(Y_{i, l}-X_{i}\right) & k_{l}^{2}\left(a_{i}^{-}\right)\left(\widetilde{\beta}^{\prime}\left(a_{i}^{+}\right)\right)^{3}\left(\left(Y_{i, l}-X_{i}\right)^{2}+\left(Y_{i, l}^{2}-X_{i}^{3}\right)\right)
\end{array}\right) .
$$

We have

$$
\operatorname{det}\left(A_{i}\left(k_{l}\right)\right)=k_{l}^{2}\left(a_{i}^{-}\right)\left(\widetilde{\beta}^{\prime}\left(a_{i}^{+}\right)\right)^{4} P_{Y_{i, l}}\left(X_{i}\right)
$$

where

$$
P_{Y_{i, l}}\left(X_{i}\right)=\left(1-X_{i}\right)\left(Y_{i, l}^{2}-X_{i}^{3}\right)-X_{i}\left(Y_{i, l}-X_{i}\right)^{2} .
$$

If $\widetilde{\beta}^{\prime}\left(a_{i}^{+}\right)>0$ (respectively, $\left.\widetilde{\beta}^{\prime}\left(a_{i}^{+}\right)<0\right)$, $\left[\widetilde{\beta}^{\prime}\right]_{a_{i}}>0$ is equivalent to $X_{i}<1$ (respectively, $X_{i}>1$ ).

Consequently, we have:

For $i \leq p$ (on the left-hand side of $\left.\omega_{0}\right)$ : (2.4) $\Longleftrightarrow 0<X_{i}<1$ and $P_{Y_{i, l}}\left(X_{i}\right)>0$,

$\forall l \in\{1, \ldots, m\}$.

For $i>p$ (on the right-hand side of $\left.\omega_{0}\right):(2.4) \Longleftrightarrow X_{i}>1$ and $P_{Y_{i, l}}\left(X_{i}\right)>0$,

$\forall l \in\{1, \ldots, m\}$. 
We assume here that the coefficients $Y_{i, l}(l \in\{1, \ldots, m\})$ cannot be smooth simultaneously at the same point (i.e., $Y_{i, l}=1 \Rightarrow Y_{i, l^{\prime}} \neq 1$ for $l \neq l^{\prime}$ and fixed $i \in\{1, \ldots, n-1\}$ ).

For the first case $(i \leq p)$, we are going to prove that

$$
X_{i}=\frac{1}{1+\max _{1 \leq j \leq m}\left\{\left|\frac{1}{Y_{i, j}}-1\right|\right\}}
$$

satisfies (2.5).

We note $L_{-}:=\left\{l \in\{1, \ldots, m\} ; Y_{l}<1\right\}, L_{+}:=\left\{l \in\{1, \ldots, m\} ; Y_{l}>1\right\}$ with $Y_{j}=Y_{i, j}$ and $X=X_{i}$. We have then the following cases:

1. $L_{-}=\emptyset$. In this case, we have $X=\frac{\max _{1 \leq j \leq m}\left\{Y_{j}\right\}}{2 \max _{1 \leq j \leq m}\left\{Y_{j}\right\}-1}$. Then, for $l, l^{\prime} \in\{1, \ldots m\}$, we obtain

$$
\begin{aligned}
Y_{l} & \geq Y_{l^{\prime}} \geq 1 \\
& \Rightarrow \quad P_{Y_{l^{\prime}}}\left(\frac{Y_{l}}{2 Y_{l}-1}\right)=\frac{Y_{l}^{2}\left(Y_{l}-1\right)^{2}}{\left(2 Y_{l}-1\right)^{4}}+\frac{\left(Y_{l}-Y_{l^{\prime}}\right)^{2}}{\left(2 Y_{l}-1\right)^{2}}+\frac{2 Y_{l}\left(Y_{l}-Y_{l^{\prime}}\right)\left(Y_{l^{\prime}}-1\right)}{\left(2 Y_{l}-1\right)^{2}}>0 .
\end{aligned}
$$

2. $L_{+}=\emptyset$. In this case, we have $X=\min _{1 \leq j \leq m}\left\{Y_{j}\right\}$ and

$$
Y_{l^{\prime}} \leq Y_{l} \leq 1 \quad \Rightarrow \quad P_{Y_{l}}\left(Y_{l^{\prime}}\right)=Y_{l^{\prime}}^{2}\left(1-Y_{l^{\prime}}\right)^{2}+\left(Y_{l}-Y_{l^{\prime}}\right)^{2}+2\left(Y_{l}-Y_{l^{\prime}}\right) Y_{l^{\prime}}\left(1-Y_{l}\right)>0 .
$$

3. $L_{-} \neq \emptyset$ and $L_{+} \neq \emptyset$. We distinguish the following cases:

(i) $l \in L_{+}\left(Y_{l}>1\right)$

(a) If $X=\frac{1}{1+\left|\frac{1}{Y_{l^{\prime}}}-1\right|}$ with $l^{\prime} \in L_{-}\left(Y_{l^{\prime}}<1\right)$ and $l \neq l^{\prime}$, then we have $X=Y_{l^{\prime}}$ and $\frac{1}{Y_{l^{\prime}}}+\frac{1}{Y_{l}} \geq 2$ with $Y_{l^{\prime}}<1<Y_{l}$ and we obtain

$$
P_{Y_{l}}\left(Y_{l^{\prime}}\right)=Y_{l^{\prime}}^{2}\left(1-Y_{l^{\prime}}\right)^{2}+\left(Y_{l}-Y_{l^{\prime}}\right)\left(Y_{l}+Y_{l^{\prime}}-2 Y_{l^{\prime}} Y_{l}\right)>0 .
$$

(b) If $X=\frac{1}{1+\left|\frac{1}{Y_{l^{\prime}}}-1\right|}$ with $l^{\prime} \in L_{+}\left(Y_{l^{\prime}}>1\right)$ and $l \neq l^{\prime}$, then we have $X=\frac{Y_{l^{\prime}}}{2 Y_{l^{\prime}}-1}$, which corresponds to the case $L_{-}=\emptyset$.

(ii) $l \in L_{-}\left(Y_{l}<1\right)$

(a) If $X=\frac{1}{1+\left|\frac{1}{Y_{l^{\prime}}}-1\right|}$ with $l^{\prime} \in L_{-}\left(Y_{l^{\prime}}<1\right)$ and $l \neq l^{\prime}$. This case is reduced to the case $L_{+}=\emptyset$.

(b) If $X=\frac{1}{1+\left|\frac{1}{Y_{l^{\prime}}}-1\right|}$ with $l^{\prime} \in L_{+}\left(Y_{l^{\prime}}>1\right)$ and $l \neq l^{\prime}$, then we obtain $X=\frac{Y_{l^{\prime}}}{2 Y_{l^{\prime}}-1}$, $\frac{1}{Y_{l^{\prime}}}+\frac{1}{Y_{l}} \leq 2$ with $Y_{l}<1<Y_{l^{\prime}}$, and

$$
P_{Y_{l}}\left(\frac{Y_{l^{\prime}}}{2 Y_{l^{\prime}}-1}\right)=\frac{Y_{l^{\prime}}^{2}\left(Y_{l^{\prime}}-1\right)^{2}}{\left(2 Y_{l^{\prime}}-1\right)^{4}}+\frac{\left(Y_{l^{\prime}}-Y_{l}\right)\left(2 Y_{l} Y_{l^{\prime}}-Y_{l^{\prime}}-Y_{l}\right)}{\left(2 Y_{l^{\prime}}-1\right)^{2}}>0
$$

So (2.5) is satisfied for the choice (2.7).

For the second case $(i>p)$, we are going to prove that

$$
X_{i}=1+\max _{1 \leq j \leq m}\left\{\left|Y_{i, j}-1\right|\right\}
$$

satisfies (2.6). We have the following cases: 
1. $L_{-}=\emptyset$. In this case, we have $X=\max _{1 \leq j \leq m}\left\{Y_{j}\right\}$. Then, for $l, l^{\prime} \in\{1, \ldots m\}$, we obtain $Y_{l} \geq Y_{l^{\prime}} \geq 1 \Rightarrow P_{Y_{l^{\prime}}}\left(Y_{l}\right)=Y_{l}^{2}\left(1-Y_{l}\right)^{2}+\left(Y_{l}-Y_{l^{\prime}}\right)^{2}+2\left(Y_{l}-Y_{l^{\prime}}\right) Y_{l}\left(Y_{l^{\prime}}-1\right)>0$.

2. $L_{+}=\emptyset$. In this case, we have $X=2-\min _{1 \leq j \leq m}\left\{Y_{j}\right\}$, and

$$
\begin{aligned}
& Y_{l^{\prime}} \leq Y_{l} \leq 1 \\
& \Rightarrow \quad P_{Y_{l}}\left(2-Y_{l^{\prime}}\right)=\left(1-Y_{l^{\prime}}\right)^{2}\left(Y_{l^{\prime}}^{2}+4\left(Y_{l}-Y_{l^{\prime}}\right)\right)+\left(Y_{l}-Y_{l^{\prime}}\right)^{2} \\
&+2\left(Y_{l}-Y_{l^{\prime}}\right)\left(1-Y_{l}\right)\left(2-Y_{l^{\prime}}\right)>0 .
\end{aligned}
$$

3. $L_{-} \neq \emptyset$ and $L_{+} \neq \emptyset$. We distinguish the following cases:

(i) $l \in L_{+}\left(Y_{l}>1\right)$

(a) If $X=1+\left|Y_{l^{\prime}}-1\right|$ with $l^{\prime} \in L_{-}\left(Y_{l^{\prime}}<1\right)$ and $l \neq l^{\prime}$, then we have $X=2-Y_{l^{\prime}}$ and $Y_{l^{\prime}}+Y_{l} \leq 2$ with $Y_{l^{\prime}}<Y_{l}<1$, thus

$$
P_{Y_{l}}\left(2-Y_{l^{\prime}}\right)=\left(1-Y_{l^{\prime}}\right)^{2} Y_{l^{\prime}}^{2}+\left(Y_{l}-Y_{l^{\prime}}\right)\left(2-Y_{l^{\prime}}-Y_{l}\right)\left(3-2 Y_{l^{\prime}}\right)+2\left(Y_{l}-Y_{l^{\prime}}\right)\left(Y_{l^{\prime}}-1\right)^{2}>0 .
$$

(b) If $X=1+\left|Y_{l^{\prime}}-1\right|$ with $l^{\prime} \in L_{+}\left(Y_{l^{\prime}}>1\right)$ and $l \neq l^{\prime}$, we obtain $X=Y_{l^{\prime}}$. This case is reduced to the case $L_{-}=\emptyset$.

(ii) $l \in L_{-}\left(Y_{l}<1\right)$

(a) If $X=1+\left|Y_{l^{\prime}}-1\right|$ with $l^{\prime} \in L_{-}\left(Y_{l^{\prime}}<1\right)$ and $l \neq l^{\prime}$. This case is reduced to the case $L_{+}=\emptyset$.

(b) If $X=1+\left|Y_{l^{\prime}}-1\right|$ with $l^{\prime} \in L_{+}\left(Y_{l^{\prime}}>1\right)$ and $l \neq l^{\prime}$, we have $X=Y_{l^{\prime}}, Y_{l^{\prime}}+Y_{l} \geq 2$ with $Y_{l^{\prime}}>1>Y_{l}$, and

$$
P_{Y_{l}}\left(Y_{l^{\prime}}\right)=\left(1-Y_{l^{\prime}}\right)^{2}\left(Y_{l^{\prime}}^{2}-2 Y_{l^{\prime}}+2 Y_{l}\right)+\left(2 Y_{l^{\prime}}-1\right)\left(Y_{l^{\prime}}-Y_{l}\right)\left(Y_{l^{\prime}}+Y_{l}-2\right)>0 .
$$

(We have used $Y_{l}+Y_{l^{\prime}} \geq 2 \Rightarrow Y_{l^{\prime}}-1 \geq 1-Y_{l} \geq 0 \Rightarrow\left(Y_{l^{\prime}}-1\right)^{2} \geq\left(1-Y_{l}\right)^{2} \Rightarrow$ $Y_{l^{\prime}}^{2}-2 Y_{l^{\prime}} \geq Y_{l}^{2}-2 Y_{l} \Rightarrow Y_{l^{\prime}}^{2}-2 Y_{l^{\prime}}+2 Y_{l} \geq Y_{l}^{2} \geq 0$.)

Then (2.6) is satisfied for the choice (2.8) and the proof of the lemma is achieved.

Remark 2.2 The case $m=1$ corresponds to the choice made in [8].

We now define the function $\beta=\widetilde{\beta}+K$ with $\widetilde{\beta}$ chosen as in the previous lemma and $K=r\|\widetilde{\beta}\|_{\infty}, r>1$. For $\lambda>0$ and $t \in(0, T)$, we define the following weight functions:

$$
\varphi(x, t)=\frac{e^{\lambda \beta(x)}}{t(T-t)}, \quad \eta(x, t)=\frac{e^{\lambda \bar{\beta}}-e^{\lambda \beta(x)}}{t(T-t)}
$$

with $\bar{\beta}=2 r\|\widetilde{\beta}\|_{\infty}$ (see $\left.[18,19]\right)$. Observe that the functions $\eta$ and $\varphi$ are positive.

We introduce

$$
\begin{aligned}
\Theta= & \left\{q \in \mathcal{C}(Q) ; q_{\left.\right|_{Q_{i}}} \in \mathcal{C}^{2}\left(\bar{Q}_{i}\right), i=0, \ldots, n-1,\right. \\
& \left.q_{\left.\right|_{\Sigma}}=0 \text { and } q \text { satisfies }(\mathrm{TC}) \text { for all } t \in(0, T)\right\},
\end{aligned}
$$

where $Q_{i}=\Omega_{i} \times(0, T)$.

We set $\psi=e^{-s \eta} q$, and let us introduce, for fixed $l \in\{1, \ldots, m\}$, the following operators:

$$
\begin{aligned}
& M_{1}^{(l)} \psi=-\partial_{x}\left(k_{l} \partial_{x} \psi\right)-s^{2} \lambda^{2} \varphi^{2}\left(\beta^{\prime}\right)^{2} k_{l} \psi+s\left(\partial_{t} \eta\right) \psi, \\
& M_{2}^{(l)} \psi=\partial_{t} \psi+2 s \lambda \varphi k_{l} \beta^{\prime} \partial_{x} \psi+2 s \lambda^{2} \varphi k_{l}\left(\beta^{\prime}\right)^{2} \psi .
\end{aligned}
$$


By applying the scalar Carleman proved in [5, Eq. (1.6)] for the operator $\partial_{t}-\partial_{x}\left(k_{l} \partial_{x}\right)$ and $q=y_{l}$, we obtain the following theorem.

Theorem 2.1 Let fixed $l \in\{1, \ldots, m\}$. We assume that the diffusion coefficient $k_{l}$ is piecewise- $\mathcal{C}^{1}(\Omega)$ and Assumption 1.1 is satisfied. Then there exist $\lambda_{0}=\lambda_{0}\left(\Omega, \omega,\left\|k_{l}\right\|_{L^{\infty}}\right) \geq 1$, $s_{0}=s_{0}\left(\lambda_{0}, T\right)>0$ and a positive constant $C_{0}=C_{0}\left(\Omega, \omega,\left\|k_{l}\right\|_{L^{\infty}}\right)$ such that, for any $\lambda \geq \lambda_{0}$ and any $s \geq s_{0}$, the following estimate holds:

$$
\begin{aligned}
& \left\|M_{1}^{(l)}\left(e^{-s \eta} y_{l}\right)\right\|_{L^{2}\left(Q^{\prime}\right)}^{2}+\left\|M_{2}^{(l)}\left(e^{-s \eta} y_{l}\right)\right\|_{L^{2}\left(Q^{\prime}\right)}^{2} \\
& \quad+s \lambda^{2} \iint_{Q} e^{-2 s \eta} \varphi\left|\partial_{x} y_{l}\right|^{2} d x d t+s^{3} \lambda^{4} \iint_{Q} e^{-2 s \eta} \varphi^{3}\left|y_{l}\right|^{2} d x d t \\
& \leq C_{0}\left[s^{3} \lambda^{4} \iint_{\omega \times(0, T)} e^{-2 s \eta} \varphi^{3}\left|y_{l}\right|^{2} d x d t+\iint_{Q} e^{-2 s \eta}\left|\partial_{t} y_{l}-\partial_{x}\left(k_{l} \partial_{x} y_{l}\right)\right|^{2} d x d t\right]
\end{aligned}
$$

for $y_{l} \in \Theta$.

Remark 2.3 Carleman estimate (2.10) remains the same if we consider the operator $\partial_{t}+$ $\partial_{x}\left(k_{l} \partial_{x}\right)$ instead of $\partial_{t}-\partial_{x}\left(k_{l} \partial_{x}\right)$.

From the above theorem, we have the following result (see $[18,19])$.

Proposition 2.1 Let fixed $l \in\{1, \ldots, m\}$. We assume that the diffusion coefficient $k_{l}$ is piecewise- $\mathcal{C}^{1}(\Omega)$ and Assumption 1.1 is satisfied. Then there exist $\lambda_{0}=\lambda_{0}\left(\Omega, \omega,\left\|k_{l}\right\|_{L^{\infty}}\right) \geq 1$, $s_{0}=s_{0}\left(\lambda_{0}, T\right)>0$ and a positive constant $C_{0}=C_{0}\left(\Omega, \omega,\left\|k_{l}\right\|_{L^{\infty}}\right)$ such that, for any $\lambda \geq \lambda_{0}$ and any $s \geq s_{0}$, the following estimate holds:

$$
\begin{aligned}
& s^{-1} \iint_{Q} e^{-2 s \eta} \varphi^{-1}\left(\left|\partial_{t} y_{l}\right|^{2}+\left|\partial_{x}\left(k_{l} \partial_{x} y_{l}\right)\right|^{2}\right) d x d t+s \lambda^{2} \iint_{Q} e^{-2 s \eta} \varphi\left|\partial_{x} y_{l}\right|^{2} d x d t \\
& \quad+s^{3} \lambda^{4} \iint_{Q} e^{-2 s \eta} \varphi^{3}\left|y_{l}\right|^{2} d x d t \\
& \leq C_{0}\left[s^{3} \lambda^{4} \iint_{\omega \times(0, T)} e^{-2 s \eta} \varphi^{3}\left|y_{l}\right|^{2} d x d t+\iint_{Q} e^{-2 s \eta}\left|\partial_{t} y_{l}-\partial_{x}\left(k_{l} \partial_{x} y_{l}\right)\right|^{2} d x d t\right]
\end{aligned}
$$

for $y_{l} \in \Theta$.

We consider the following functional:

$$
\begin{aligned}
I\left(k_{l}, y_{l}\right)= & s^{-1} \iint_{Q} e^{-2 s \eta} \varphi^{-1}\left(\left|\partial_{t} y_{l}\right|^{2}+\left|\partial_{x}\left(k_{l} \partial_{x} y_{l}\right)\right|^{2}\right) d x d t \\
& +s \lambda^{2} \iint_{Q} e^{-2 s \eta} \varphi\left|\partial_{x} y_{l}\right|^{2} d x d t+s^{3} \lambda^{4} \iint_{Q} e^{-2 s \eta} \varphi^{3}\left|y_{l}\right|^{2} d x d t, \quad l=1, \ldots, m .
\end{aligned}
$$

Using the previous proposition, we have the following theorem.

Theorem 2.2 Let $j, k=1, \ldots, m$ and $M=\sum_{k=1}^{m} \max _{1 \leq j \leq m}\left\|a_{j k}\right\|_{\infty}^{2}$ with $a_{j k} \in L^{\infty}(Q)$. We assume that the diffusion coefficients $k_{j}$ are piecewise- $\mathcal{C}^{1}(\Omega)$ and satisfy Assumption 1.1. 
Then there exist $\lambda_{0}=\lambda_{0}\left(\Omega, \omega,\left\|k_{j}\right\|_{L^{\infty}}\right) \geq 1, s_{1}=s_{1}\left(\lambda_{0}, T, M\right)>0$ and a positive constant $C_{1}=C_{1}\left(\Omega, \omega,\left\|k_{j}\right\|_{L^{\infty}}\right)$ such that, for any $\lambda \geq \lambda_{0}$ and any $s \geq s_{1}$, the following estimate holds:

$$
\sum_{j=1}^{m} I\left(k_{j}, y_{j}\right) \leq C_{1} \sum_{j=1}^{m}\left[s^{3} \lambda^{4} \int_{0}^{T} \int_{\omega} e^{-2 s \eta} \varphi^{3}\left|y_{j}\right|^{2} d x d t+\iint_{Q} e^{-2 s \eta}\left|f_{j}\right|^{2} d x d t\right]
$$

for any solution $y=\left(y_{j}\right)_{1 \leq j \leq m}$ of $(1.1)$.

Proof Observing that there exists $C_{2}=C_{2}(\Omega, \omega)$ such that $1 \leq C_{2} \frac{T^{6}}{4^{3}} \varphi^{3}$, by adding estimates (2.11) for $l=1, \ldots, m$, we obtain

$$
\begin{aligned}
\sum_{j=1}^{m} I\left(k_{j}, y_{j}\right) \leq & C_{1} \sum_{j=1}^{m}\left[s^{3} \lambda^{4} \int_{0}^{T} \int_{\omega} e^{-2 s \eta} \varphi^{3}\left|y_{j}\right|^{2} d x d t+\iint_{Q} e^{-2 s \eta}\left|f_{j}\right|^{2} d x d t\right] \\
& +C_{3} \sum_{j=1}^{m} \iint_{Q} e^{-2 s \eta} \varphi^{3}\left|y_{j}\right|^{2} d x d t
\end{aligned}
$$

with

$$
C_{1}=2^{m} C_{0} \quad \text { and } \quad C_{3}=C_{1} C_{2} \frac{T^{6}}{4^{3}} M
$$

Choosing then

$$
s \geq s_{1}=\left(\frac{C_{3}}{\lambda_{0}{ }^{4}}\right)^{\frac{1}{3}}
$$

the last term on the right-hand side of (2.13) can be 'absorbed' by the terms in $\sum_{j=1}^{m} I\left(k_{j}, y_{j}\right)$. This concludes the proof.

\section{Remark 2.4}

1. Carleman estimate (2.12) remains valid if we consider the boundary observation $\gamma=\{0\}$ (respectively $\gamma=\{1\}$ ) instead of the interior observation $\omega$. The result is obtained through a modified form of Lemma 2.1, namely:

Modified Lemma 2.1 There exists a function $\widetilde{\beta} \in \mathcal{C}(\bar{\Omega})$ such that

$$
\begin{aligned}
& \left.\widetilde{\beta}\right|_{\Omega_{i}} \in \mathcal{C}^{2}\left(\Omega_{i}\right), \quad i=0, \ldots, n-1, \\
& \widetilde{\beta}>0 \quad \text { in } \Omega, \quad \widetilde{\beta}(1)=0 \quad(\text { respectively } \widetilde{\beta}(0)=0), \\
& \left.\left.\widetilde{\beta}^{\prime}\right|_{\left[a_{p}, a_{p+1}\right]} \leq \vartheta<0 \quad \text { (respectively }\left.\widetilde{\beta}^{\prime}\right|_{\left[a_{p}, a_{p+1}\right]} \geq \vartheta>0\right)
\end{aligned}
$$

and the function $\widetilde{\beta}$ satisfies the following trace properties, $\forall l \in\{1, \ldots, m\}$ and some $\alpha>0$,

$$
\forall\left(a_{i}, k_{l}\right) \in \Lambda, \quad\left(A_{i}\left(k_{l}\right) u, u\right) \geq \alpha\|u\|^{2}, \quad i=1, \ldots, n-1,
$$

with $u=\left(u_{1}, u_{2}\right)^{t}, u_{1}, u_{2} \in \mathbb{R}$ and the matrices $A_{i}\left(k_{l}\right)$ are defined by

$$
A_{i}\left(k_{l}\right)=\left(\begin{array}{cc}
{\left[\widetilde{\beta}^{\prime}\right]_{a_{i}}} & \widetilde{\beta}^{\prime}\left(a_{i}^{+}\right)\left[k_{l} \widetilde{\beta}^{\prime}\right]_{a_{i}} \\
\widetilde{\beta}^{\prime}\left(a_{i}^{+}\right)\left[k_{l} \widetilde{\beta}^{\prime}\right]_{a_{i}} & \widetilde{\beta}^{\prime}\left(a_{i}^{+}\right)\left[k_{l} \widetilde{\beta}^{\prime}\right]_{a_{i}}^{2}+\left[k_{l}^{2}\left(\widetilde{\beta}^{\prime}\right)^{3}\right]_{a_{i}}
\end{array}\right), \quad i=1, \ldots, n-1 .
$$


2. By inspecting the proof of Theorem 2.10 (see [5, Remark 1.4(5)]), we observe that we can obtain the same Carleman estimate (2.12) which incorporates estimates of the traces of $y_{j}$ and $\partial_{x} y_{j}, j=1, \ldots, m$.

\section{Global Carleman estimate with ' $m$ observations' in the case of $B V$ diffusion coefficients}

In this section, we generalize the Carleman estimate given in [8] to a parabolic system using the results obtained in the previous section. We show that we can prove the global Carleman estimate in the case of bounded variations $(B V)$ diffusion coefficients for a system of $m$ coupled parabolic equations with an interior observation region $\omega \times(0, T)$, where $\omega$ is a non-empty open subset of $\Omega$. We follow the method developed in [8] and many notations and arguments of the previous paper will be reproduced here.

We consider system (1.1) with diffusion coefficients $k_{j}$ assumed here to be of $B V$ such that $k_{j}$ are of class $\mathcal{C}^{1}$ on $\bar{\omega}$ and satisfy Assumption 1.1.

Our goal is to construct a limit weight function $\beta$ (the same for each equation) using the approach of $B V$ diffusion coefficients by piecewise-constant coefficients. This process allows us to derive a Carleman estimate for the operator $\partial_{t}+\mathcal{A}$.

Let $\omega_{0} \Subset \omega \Subset \Omega$. Without any loss of generality, we suppose that $\omega=\left(x_{0}, x_{1}\right)$ with $0<$ $x_{0}<x_{1}<1$. We denote the total variations of $k_{j}$ on $\left[0, x_{0}\right]$ and $\left[x_{1}, 1\right]$ by $V_{0}^{j}=V_{0}^{x_{0}}\left(k_{j}\right)$ and $V_{1}^{j}=V_{x_{1}}^{1}\left(k_{j}\right)$.

Let $\varepsilon>0$. There exist functions $k_{j, \varepsilon}>0$, piecewise-constant on $\left(0, x_{0}\right) \cup\left(x_{1}, 1\right)$ and smooth on $\omega$, such that for any $j=1, \ldots, m$ (see $[20]$ ),

$$
\begin{aligned}
& \left\|k_{j}-k_{j, \varepsilon}\right\|_{L^{\infty}(\Omega)} \leq \varepsilon, \quad V_{0}^{x_{0}}\left(k_{j, \varepsilon}\right) \leq V_{0}^{j} \\
& V_{x_{1}}^{1}\left(k_{j, \varepsilon}\right) \leq V_{1}^{j} \quad \text { and } \quad\left\|k_{j}-k_{j, \varepsilon}\right\|_{\mathcal{C}^{1}(\bar{\omega})} \leq \varepsilon
\end{aligned}
$$

We consider the points $a_{i}(1 \leq i \leq n)$ in the interval $\left[0, x_{0}\right]$ such that $\left(a_{i}, k_{j, \varepsilon}\right) \in \Lambda$.

We note

$$
Y_{i, j}^{\varepsilon}=\frac{k_{j, \varepsilon}\left(a_{i}^{+}\right)}{k_{j, \varepsilon}\left(a_{i}^{-}\right)} .
$$

In the case where we are on the left-hand side of $\omega_{0}(i \leq p)$, we consider the following choice (see the proof of Lemma 2.1):

$$
X_{i}^{\varepsilon}=\frac{1}{1+\max _{1 \leq j \leq m}\left\{\left|\frac{1}{Y_{i, j}^{\varepsilon}}-1\right|\right\}} .
$$

We build the piecewise-constant function $\Pi_{0}^{\varepsilon}$ as

$$
\Pi_{0}^{\varepsilon}(x):=\Pi_{0}^{\varepsilon}(0) \prod_{x>a_{l}} \frac{1}{X_{l}^{\varepsilon}}, \quad x \notin\left\{a_{1}, \ldots, a_{n}\right\}
$$

for some fixed $\Pi_{0}^{\varepsilon}(0)>0$. Observe that $X_{i}^{\varepsilon}=\frac{\Pi_{0}^{\varepsilon}\left(a_{i}^{-}\right)}{\Pi_{0}^{\varepsilon}\left(a_{i}^{+}\right)}$and $X_{i}^{\varepsilon}<1,1 \leq i \leq n$.

In a similar manner, we consider the points $a_{i}(n+1 \leq i \leq n+\varrho)$ in the interval $\left[x_{1}, 1\right]$ such that $\left(a_{i}, k_{j, \varepsilon}\right) \in \Lambda$. 
Then, in the case of the right-hand side of $\omega_{0}(i>p)$, we choose

$$
X_{i}^{\varepsilon}=1+\max _{1 \leq j \leq m}\left\{\left|Y_{i, j}^{\varepsilon}-1\right|\right\} .
$$

We construct now the piecewise-constant function $\Pi_{1}^{\varepsilon}$ as

$$
\Pi_{1}^{\varepsilon}(x):=\Pi_{1}^{\varepsilon}(1) \prod_{x<a_{l}} X_{l}^{\varepsilon}, \quad x \notin\left\{a_{n+1}, \ldots, a_{n+\varrho}\right\}
$$

for some fixed $\Pi_{1}^{\varepsilon}(1)<0$. Observe that $X_{i}^{\varepsilon}=\frac{\Pi_{1}^{\varepsilon}\left(a_{i}^{-}\right)}{\Pi_{1}^{\varepsilon}\left(a_{i}^{+}\right)}$and $X_{i}^{\varepsilon}>1, n+1 \leq i \leq n+\varrho$.

Now, we define the functions $\widetilde{\beta}_{0, \varepsilon}(x):=\int_{0}^{x} \Pi_{0}^{\varepsilon}(y) d y$ and $\widetilde{\beta}_{1, \varepsilon}(x):=\int_{1}^{x} \Pi_{1}^{\varepsilon}(y) d y$. Thus we define a continuous function $\widetilde{\beta}_{\varepsilon}$ as follows:

$$
\widetilde{\beta}_{\varepsilon}(x)= \begin{cases}\widetilde{\beta}_{0, \varepsilon}(x) & \text { in }\left[0, x_{0}\right], \\ \widetilde{\beta}_{1, \varepsilon}(x) & \text { in }\left[x_{1}, 1\right],\end{cases}
$$

and we design $\widetilde{\beta}_{\varepsilon}$ to be of class $\mathcal{C}^{2}$ on $\bar{\omega}$.

It is easy to see that $\widetilde{\beta}_{\varepsilon}$ satisfies the conditions listed in Lemma 2.1. Then Carleman estimate (2.11) remains valid for the operators $\partial_{t}-\partial_{x}\left(k_{j, \varepsilon} \partial_{x}\right), j=1, \ldots, m$, with the associated weight functions $\varphi_{\varepsilon}, \eta_{\varepsilon}$. Hence, we introduce

$$
\beta_{\varepsilon}=\widetilde{\beta}_{\varepsilon}+K_{\varepsilon}
$$

with $K_{\varepsilon}=r\left\|\widetilde{\beta}_{\varepsilon}\right\|_{\infty}, r>1$. For $\lambda>0$ and $t \in(0, T)$, we define the following weight functions:

$$
\varphi_{\varepsilon}(x, t)=\frac{e^{\lambda \beta_{\varepsilon}(x)}}{t(T-t)}, \quad \eta_{\varepsilon}(x, t)=\frac{e^{\lambda \bar{\beta}_{\varepsilon}}-e^{\lambda \beta_{\varepsilon}(x)}}{t(T-t)}
$$

with $\bar{\beta}_{\varepsilon}=2 r\left\|\widetilde{\beta_{\varepsilon}}\right\|_{\infty}$.

In this section, we want to pass to the limit in Carleman estimate (2.11). We first need to control the behavior of the derivative of $\beta_{\varepsilon}$ as $\varepsilon$ goes to zero. This is the object of the following lemma.

Lemma 3.1 (see [8, Lemma 3.2]) Let $j=1, \ldots, m$. We assume that the diffusion coefficients $k_{j} \in B V(\Omega)$ and Assumption 1.1 is satisfied, then there exist $\varepsilon_{0}>0, K_{0}=K_{0}\left(k_{j, \min }, \varepsilon_{0}\right)>0$ and $K_{1}=K_{1}\left(k_{j, \min }, \varepsilon_{0}\right)>0$ such that, for all $0<\varepsilon \leq \varepsilon_{0} \leq \min _{1 \leq j \leq m}\left(k_{j, \min }\right), V_{0}^{x_{0}}\left(\Pi_{0}^{\varepsilon}\right) \leq$ $K_{0} \Pi_{0}^{\varepsilon}(0)$ and $V_{x_{1}}^{1}\left(\Pi_{1}^{\varepsilon}\right) \leq K_{1}\left|\Pi_{1}^{\varepsilon}(1)\right|$.

Using Helly's theorem (see [20]), the function $\Pi_{0}^{\varepsilon}$ (respectively $\Pi_{1}^{\varepsilon}$ ) converges everywhere to the function $\Pi_{0}$ (respectively $\Pi_{1}$ ) as $\varepsilon$ goes to 0 . Since the function $\Pi_{0}^{\varepsilon}$ (respectively $\Pi_{1}^{\varepsilon}$ ) is bounded in $L^{\infty}\left(0, x_{0}\right)$ (respectively in $\left.L^{\infty}\left(x_{1}, 1\right)\right)$ uniformly with respect to $\varepsilon$, we deduce, by applying the dominated convergence theorem and Lemma 3.1, that the function $\widetilde{\beta}_{0, \varepsilon}$ (respectively $\widetilde{\beta}_{1, \varepsilon}$ ) converges everywhere to the function $\widetilde{\beta}_{0}(x):=\int_{0}^{x} \Pi_{0}(y) d y$ (respectively $\widetilde{\beta}_{1}(x):=\int_{1}^{x} \Pi_{1}(y) d y$ ).

Then we can define the function $\widetilde{\beta}$ on $\Omega$ as follows :

$$
\widetilde{\beta}(x)= \begin{cases}\widetilde{\beta}_{0}(x) & \text { in }\left[0, x_{0}\right], \\ \widetilde{\beta}_{1}(x) & \text { in }\left[x_{1}, 1\right],\end{cases}
$$


and $\widetilde{\beta}, \widetilde{\beta}_{\varepsilon}$ are of a class $\mathcal{C}^{2}$ on $\bar{\omega}$ and satisfy the following properties:

1. $\widetilde{\beta}_{\varepsilon}$ converges everywhere to $\widetilde{\beta}$ in $\mathcal{C}(\bar{\Omega})$.

2. $\widetilde{\beta}_{\varepsilon \mid \omega}$ converges to $\widetilde{\beta}_{\mid \omega}$ in $\mathcal{C}^{2}(\bar{\omega})$.

3. $\left|\widetilde{\beta_{\varepsilon}^{\prime}}(x)\right| \geq \min \left(\widetilde{\beta}^{\prime}(0),\left|\widetilde{\beta}^{\prime}(1)\right|\right)$ and $\left|\widetilde{\beta}^{\prime}(x)\right| \geq \min \left(\widetilde{\beta}^{\prime}(0),\left|\widetilde{\beta}^{\prime}(1)\right|\right)$.

Hence, we introduce

$$
\beta=\widetilde{\beta}+K
$$

with $K=r\|\widetilde{\beta}\|_{\infty}, r>1$. For $\lambda>0$ and $t \in(0, T)$, we define the following weight functions:

$$
\varphi(x, t)=\frac{e^{\lambda \beta(x)}}{t(T-t)}, \quad \eta(x, t)=\frac{e^{\lambda \bar{\beta}}-e^{\lambda \beta(x)}}{t(T-t)}
$$

with $\bar{\beta}=2 r\|\widetilde{\beta}\|_{\infty}$.

From the above arguments, we obtain the following lemma.

Lemma 3.2 (see [8, Lemma 3.3]) Let $j=1, \ldots, m$. We assume that $k_{j}$ in $B V(\Omega)$ is of class $\mathcal{C}^{1}$ in $\bar{\omega}$ and satisfies Assumption 1.1. Let $k_{j, \varepsilon}$ be piecewise-constant on $\Omega \backslash \omega$ and smooth on $\omega$ such that (3.1) is satisfied. Then there exists a function $\widetilde{\beta}_{\varepsilon}$ that satisfies the properties listed in Lemma 2.1 for the associated coefficients $k_{j, \varepsilon}$. Furthermore, $\widetilde{\beta}$ and $\widetilde{\beta}_{\varepsilon}$ are of class $\mathcal{C}^{2}$ on $\bar{\omega}$ and satisfy the above properties $(1,2,3)$.

Remark 3.1 The results obtained in Lemma 3.2 imply that the constants $\overline{\beta_{\varepsilon}}$ and $K_{\varepsilon}$ can now be chosen uniformly with respect to $\varepsilon$.

Under the same assumptions as in Lemma 3.2 and the properties of $\widetilde{\beta}$ and $\widetilde{\beta}_{\varepsilon}$ defined as above, we obtain the following proposition.

Proposition 3.1 (see [8, Proposition 3.4]) Let fixed $l \in\{1, \ldots, m\}$. Then the constant $C_{0}$ on the right-hand side of Carleman estimate (2.11) for the operator $\partial_{t}-\partial_{x}\left(k_{l, \varepsilon} \partial_{x}\right)$ and the constants $s_{0}$ and $\lambda_{0}$ can be chosen uniformly with respect to $\varepsilon$ for $0<\varepsilon \leq \varepsilon_{0} \leq k_{l, \min }$.

The proof of Proposition (3.1) is established through the following lemmata.

Lemma 3.3 Let fixed $l \in\{1, \ldots, m\}$. There exists $C>0$ uniform with respect to $\varepsilon \leq \varepsilon_{0} \leq$ $k_{l, \text { min }}$ such that

$$
\begin{aligned}
2 s \lambda & \sum_{i=0}^{n} \int_{0}^{T} \varphi_{\varepsilon}\left(a_{i}, t\right)\left(\left[\beta_{\varepsilon}^{\prime}\left|k_{l, \varepsilon} \partial_{x} \psi_{\varepsilon}\right|^{2}(\cdot, t)\right]_{a_{i}}+\left[k_{l, \varepsilon}^{2}\left(\beta_{\varepsilon}^{\prime}\right)^{3}\right]_{a_{i}}\left|s \lambda \varphi_{\varepsilon}\left(a_{i}, t\right) \psi_{\varepsilon}\left(a_{i}, t\right)\right|^{2}\right) d t \\
\geq & C s^{3} \lambda^{3} \sum_{i=1}^{n-1}\left(\max _{1 \leq j \leq m}\left\{\left|Y_{i, j}^{\varepsilon}-1\right|\right\}\right) \int_{0}^{T} \varphi_{\varepsilon}^{3}\left(a_{i}, t\right)\left|\psi_{\varepsilon}\left(a_{i}, t\right)\right|^{2} d t \\
& +C s \lambda \sum_{i=1}^{n-1}\left(\max _{1 \leq j \leq m}\left\{\left|Y_{i, j}^{\varepsilon}-1\right|\right\}\right) \int_{0}^{T} \varphi_{\varepsilon}\left(a_{i}, t\right)\left|\left(k_{l, \varepsilon} \partial_{x} \psi_{\varepsilon}\right)\left(a_{i}^{-}, t\right)\right|^{2} d t .
\end{aligned}
$$


Lemma 3.4 Let fixed $l \in\{1, \ldots, m\}$. Let $\sigma>0$. There exists $C_{\sigma}>0$ uniform with respect to $\varepsilon \leq \varepsilon_{0} \leq k_{l, \min }$ such that

$$
\begin{aligned}
\left|I_{1}\right|+\left|I_{2}\right|+\left|I_{3}\right| \leq & C_{\sigma}\left(s \lambda T^{3}+s \lambda^{3} T^{4}+\left(\lambda+\lambda^{3}\right) s^{2} T^{2}\right) \\
& \times \sum_{i=1}^{n-1}\left(\max _{1 \leq j \leq m}\left\{\left|Y_{i, j}^{\varepsilon}-1\right|\right\}\right) \int_{0}^{T} \varphi_{\varepsilon}^{3}\left(a_{i}, t\right)\left|\psi_{\varepsilon}\left(a_{i}, t\right)\right|^{2} d t \\
& +s \lambda \sigma \sum_{i=1}^{n-1}\left(\max _{1 \leq j \leq m}\left\{\left|Y_{i, j}^{\varepsilon}-1\right|\right\}\right) \int_{0}^{T} \varphi_{\varepsilon}\left(a_{i}, t\right)\left|\left(k_{l, \varepsilon} \partial_{x} \psi_{\varepsilon}\right)\left(a_{i}^{-}, t\right)\right|^{2} d t,
\end{aligned}
$$

with

$$
\begin{aligned}
& I_{1}=-\frac{1}{2} s \lambda \sum_{i=1}^{n-1} \int_{0}^{T} \partial_{t} \varphi_{\varepsilon}\left(a_{i}, t\right)\left[k_{l, \varepsilon} \beta_{\varepsilon}^{\prime}\right]_{a_{i}}\left|\psi_{\varepsilon}\left(a_{i}, t\right)\right|^{2} d t, \\
& I_{2}=2 s \lambda^{2} \sum_{i=1}^{n-1} \int_{0}^{T} \varphi_{\varepsilon}\left(a_{i}, t\right) \psi_{\varepsilon}\left(a_{i}, t\right)\left[k_{l, \varepsilon}^{2}\left(\beta_{\varepsilon}^{\prime}\right)^{2} \partial_{x} \psi_{\varepsilon}(\cdot, t)\right]_{a_{i}} d t, \\
& I_{3}=-s^{2} \lambda \sum_{i=1}^{n-1} \int_{0}^{T} \varphi_{\varepsilon}\left(a_{i}, t\right)\left(\partial_{t} \eta_{\varepsilon}\right)\left(a_{i}, t\right)\left[k_{l, \varepsilon} \beta_{\varepsilon}^{\prime}\right]_{a_{i}}\left|\psi_{\varepsilon}\left(a_{i}, t\right)\right|^{2} d t .
\end{aligned}
$$

Remark 3.2 The proofs of Lemmata 3.3 and 3.4 can be easily adapted from the proofs of Lemmata [8, Lemma 3.6] and [8, Lemma 3.5].

Following [8], we are going now pass to the limit for each term in Carleman estimate (2.12) that holds for the operator $\partial_{t}-\partial_{x}\left(k_{j, \varepsilon} \partial_{x}\right)$ as $\left\|k_{j, \varepsilon}-k_{j}\right\|_{L^{\infty}}$ goes to zero.

We recall the weight functions

$$
\varphi(x, t)=\frac{e^{\lambda \beta(x)}}{t(T-t)}, \quad \eta(x, t)=\frac{e^{\lambda \bar{\beta}}-e^{\lambda \beta(x)}}{t(T-t)},
$$

where $\beta$ is the function defined by (3.7).

Initially, we consider $f_{j} \in \mathcal{C}^{1}\left([0, T] ; L^{2}(\Omega)\right)$ with $f_{j}(0) \in H_{0}^{1}(\Omega)$ and $y_{0, j}, y_{0, j, \varepsilon} \in H_{0}^{1}(\Omega)$. Let us consider $y_{j}$ the weak solution of the system

$$
\begin{cases}\partial_{t} y+\mathcal{A} y=L y+F & \text { in } Q, \\ y(x, t)=0 & \text { on } \Sigma, \\ y(x, 0)=y_{0}(x) & \text { in } \Omega,\end{cases}
$$

and $y_{j, \varepsilon}$ the weak solution of the following system:

$$
\begin{cases}\partial_{t} y_{\varepsilon}+\mathcal{A}_{\varepsilon} y_{\varepsilon}=L y_{\varepsilon}+F & \text { in } Q, \\ y_{\varepsilon}(x, t)=0 & \text { on } \Sigma, \\ y_{\varepsilon}(x, 0)=y_{0, \varepsilon}(x) & \text { in } \Omega,\end{cases}
$$


where

$$
\mathcal{A}_{\varepsilon}=\left(\begin{array}{cccc}
\mathcal{A}_{1, \varepsilon} & 0 & \cdots & 0 \\
0 & \mathcal{A}_{2, \varepsilon} & & \vdots \\
\vdots & & \ddots & 0 \\
0 & \ldots & 0 & \mathcal{A}_{m, \varepsilon}
\end{array}\right),
$$

with $\mathcal{A}_{j, \varepsilon}=-\partial_{x}\left(k_{j, \varepsilon} \partial_{x}\right)$ in $L^{2}(\Omega), y_{\varepsilon}=\left(y_{j, \varepsilon}\right)_{1 \leq j \leq m}, y_{0, \varepsilon}=\left(y_{0, j, \varepsilon}\right)_{1 \leq j \leq m}$.

We suppose that $\partial_{x}\left(k_{j} \partial_{x} y_{0, j}\right)=\partial_{x}\left(k_{j, \varepsilon} \partial_{x} y_{0, j, \varepsilon}\right)=\mu_{j} \in H_{0}^{1}(\Omega)$. Then we can have the following inequality:

$$
\sum_{j=1}^{m}\left\|y_{0, j}-y_{0, j, \varepsilon}\right\|_{H_{0}^{1}(\Omega)}^{2} \leq C \sum_{j=1}^{m}\left\|k_{j, \varepsilon}-k_{j}\right\|_{\infty}^{2}\left\|\mu_{j}\right\|_{L^{2}(\Omega)}^{2} .
$$

Lemma 3.5 Let $t \in[0, T]$. We assume that the diffusion coefficients $k_{j}$ satisfy Assumption 1.1. Then there exists a positive constant $C$ such that the solutions to systems (3.9) and (3.10) satisfy

$$
\begin{gathered}
\sum_{j=1}^{m}\left[\left\|y_{j}(\cdot, t)-y_{j, \varepsilon}(\cdot, t)\right\|_{L^{2}(\Omega)}^{2}+\left\|\partial_{x} y_{j}-\partial_{x} y_{j, \varepsilon}\right\|_{L^{2}(Q)}^{2}\right] \\
\quad \leq C \sum_{j=1}^{m}\left\|k_{j, \varepsilon}-k_{j}\right\|_{\infty}^{2}\left(\left\|\mu_{j}\right\|_{L^{2}(\Omega)}^{2}+\left\|f_{j}\right\|_{L^{2}(Q)}^{2}\right)
\end{gathered}
$$

and

$$
\begin{aligned}
& \sum_{j=1}^{m}\left[\left\|\partial_{t} y_{j}(\cdot, t)-\partial_{t} y_{j, \varepsilon}(\cdot, t)\right\|_{L^{2}(\Omega)}^{2}+\left\|\partial_{x}\left(k_{j} \partial_{x} y_{j}\right)(\cdot, t)-\partial_{x}\left(k_{j, \varepsilon} \partial_{x} y_{j, \varepsilon}\right)(\cdot, t)\right\|_{L^{2}(\Omega)}^{2}\right] \\
& \quad \leq C \sum_{j=1}^{m}\left\|k_{j, \varepsilon}-k_{j}\right\|_{\infty}^{2}\left(\left\|\mu_{j}\right\|_{L^{2}(\Omega)}^{2}+\left\|f_{j}(0)\right\|_{L^{2}(\Omega)}^{2}+\left\|\partial_{t} f_{j}\right\|_{L^{2}(Q)}^{2}\right) .
\end{aligned}
$$

Proof Following the same steps given in the proof of Lemma 3.7 in [8], we obtain the following combination of weak formulations to systems (3.9) and (3.10):

$$
\begin{aligned}
& \sum_{j=1}^{m} \int_{\Omega}\left[\phi_{j} \partial_{t}\left(y_{j}-y_{j, \varepsilon}\right)+k_{j, \varepsilon} \partial_{x} \phi_{j} \partial_{x}\left(y_{j}-y_{j, \varepsilon}\right) d x\right] \\
& \quad=\sum_{j=1}^{m} \int_{\Omega}\left[\sum_{\rho=1}^{m} a_{j \rho}\left(y_{\rho}-y_{\rho, \varepsilon}\right) \phi_{j}+\left(k_{j, \varepsilon}-k_{j}\right) \partial_{x} y_{j} \partial_{x} \phi_{j} d x\right], \quad \phi_{j} \in L^{2}\left(0, T ; H_{0}^{1}(\Omega)\right) .
\end{aligned}
$$


Taking $\phi_{j}=y_{j}-y_{j, \varepsilon}$ and integrating over $(0, t)$, we obtain

$$
\begin{aligned}
& \frac{1}{2} \sum_{j=1}^{m}\left[\left\|y_{j}(t)-y_{j, \varepsilon}(t)\right\|_{L^{2}(\Omega)}^{2}+\left(k_{j, \min }-\sigma\right)\left\|\partial_{x} y_{j}-\partial_{x} y_{j, \varepsilon}\right\|_{L^{2}(Q)}^{2}\right] \\
& \quad \leq\left(C_{\sigma}+T\right) \sum_{j=1}^{m}\left\|k_{j, \varepsilon}-k_{j}\right\|_{\infty}^{2}\left\|\partial_{x} y_{j}\right\|_{L^{2}(Q)}^{2}+\left(\frac{1}{2}+T\right) \sum_{j=1}^{m}\left\|y_{0, j}-y_{0, j, \varepsilon}\right\|_{L^{2}(\Omega)}^{2}
\end{aligned}
$$

The previous estimate holds through the Young and Gronwall inequalities.

Observing that

$$
\begin{aligned}
& \left.\left|\iint_{Q} e^{-2 s \eta} \varphi^{3}\right| y_{j}\right|^{2} d x d t-\iint_{Q} e^{-2 s \eta_{\varepsilon}} \varphi_{\varepsilon}^{3}\left|y_{j, \varepsilon}\right|^{2} d x d t \mid \\
& \leq \iint_{Q}\left|e^{-2 s \eta} \varphi^{3}-e^{-2 s \eta_{\varepsilon}} \varphi_{\varepsilon}^{3}\right|\left|y_{j, \varepsilon}\right|^{2} d x d t \\
& \quad+\iint_{Q} e^{-2 s \eta_{\varepsilon}} \varphi_{\varepsilon}^{3}\left|y_{j}-y_{j, \varepsilon}\right|\left|y_{j}+y_{j, \varepsilon}\right| d x d t .
\end{aligned}
$$

We recall that $\beta_{\varepsilon}$ converges everywhere to $\beta$ implies that $e^{-2 s \eta_{\varepsilon}}$ and $\varphi_{\varepsilon}$ converge everywhere to $e^{-2 s \eta}$ and $\varphi$. Then, using Lemma 3.5, the Cauchy-Schwarz inequality and dominated convergence, the left-hand side of (3.14) converges to zero as $\varepsilon$ goes as zero. We obtain the same result for the remaining terms in Carleman estimate (2.12).

In conclusion, using density arguments, we obtain the following theorem.

Theorem 3.1 Let $j, k=1, \ldots, m$ and $M=\sum_{k=1}^{m} \max _{1 \leq j \leq m}\left\|a_{j k}\right\|_{\infty}^{2}$ with $a_{j k} \in L^{\infty}(Q)$. We assume that the diffusion coefficients $k_{j}$ are in $B V(\Omega)$ such that $k_{j}$ are of class $\mathcal{C}^{1}$ in $\bar{\omega}$ and satisfy Assumption 1.1. Then there exist $\lambda_{0}=\lambda_{0}\left(\Omega, \omega,\left\|k_{j}\right\|_{L^{\infty}}\right) \geq 1, s_{1}=s_{1}\left(\lambda_{0}, T, M\right)>0$ and a positive constant $C_{1}=C_{1}\left(\Omega, \omega,\left\|k_{j}\right\|_{L^{\infty}}, M\right)$ such that, for any $\lambda \geq \lambda_{0}$ and any $s \geq s_{1}$, the following estimate holds:

$$
\sum_{j=1}^{m} I_{j}\left(k_{j}, y_{j}\right) \leq C_{1} \sum_{j=1}^{m}\left[s^{3} \lambda^{4} \int_{0}^{T} \int_{\omega} e^{-2 s \eta} \varphi^{3}\left|y_{j}\right|^{2} d x d t+\iint_{Q} e^{-2 s \eta}\left|f_{j}\right|^{2} d x d t\right]
$$

for any solution $y_{j}$ of (1.1).

Remark 3.3 Carleman estimate (3.15) remains valid if we consider the boundary observation $\gamma=\{0\}$ (respectively $\gamma=\{1\}$ ) instead of the interior observation $\omega$ (see Remark 2.4). However, in this case, the assumption which corresponds to the fact that the coefficients $k_{j}$ are of class $\mathcal{C}^{1}$ in $\bar{\omega}$ is not needed to obtain (3.15).

\section{Comments and applications}

We will finalize this paper with some remarks and by establishing some additional results.

1. In the case of piecewise- $\mathcal{C}^{1}$ diffusion coefficients, many choices can be considered instead of choices (2.7) and (2.8) in the proof of Lemma 2.1. As an example, we consider

$$
X_{i}=\frac{1}{2+\sum_{j=1}^{m}\left|\frac{1}{Y_{i, j}}-1\right|}
$$


in the case $(i \leq p)$ and

$$
X_{i}=2+\sum_{j=1}^{m}\left|Y_{i, j}-1\right|
$$

in the case $(i>p)$.

For the above choices, the situation $Y_{i, l}=Y_{i, l^{\prime}}=1$ for $l \neq l^{\prime}$ and fixed $i \in\{1, \ldots, n-1\}$ becomes possible, and thus we can also obtain a global Carleman estimate in the case of smooth coefficients $k_{j}\left(\right.$ i.e., $k_{j} \in \mathcal{C}^{1}(\bar{\Omega})$ ) that holds for all $y_{j} \in \mathcal{C}^{2}(\bar{Q})$.

2. Choices (2.7) and (2.8) are taken in an optimal way in order to control the behavior of the function $\beta_{\varepsilon}$ (see Lemma (3.1)). For example, choices (4.1) and (4.2) are not appropriate in the case of $B V$ diffusion coefficients.

3. Using the results (Carleman estimate) obtained in the previous section, we deduce an observability inequality which yields null controllability. The proofs of such results can be adapted from the techniques used in [18] (also see the references therein). Consequently, we only highlight the main points.

Let us consider the following system:

$$
\begin{cases}\partial_{t} y+\mathcal{A} y=L y+V \chi_{\omega} & \text { in } Q, \\ y(x, 0)=y_{0}(x) & \text { in } \Omega,\end{cases}
$$

where $\chi_{\omega}$ is the characteristic function of the non-empty set $\omega$. The diffusion coefficients $k_{j}(j=1, \ldots, m)$ are assumed to be $B V$ such that $k_{j}$ are of class $\mathcal{C}^{1}$ in $\bar{\omega}$ and satisfy Assumption 1.1. We also assume that $a_{j k} \in L^{\infty}(Q), 1 \leq j, k \leq m, y_{0} \in H, 1 \leq j \leq m$, and the controls $v_{j} \in L^{2}(Q)$. We have also $y(\cdot, t)=\left(y_{j}(\cdot, t)\right)_{1 \leq j \leq m} \in D(\mathcal{A})$ for all $t \in(0, T)$ and $V=\left(v_{j}\right)_{1 \leq j \leq m} \in\left(L^{2}(Q)\right)^{m}$.

In order to obtain an observability inequality for system (4.3), we will consider the socalled adjoint problem of the form

$$
\begin{cases}-\partial_{t} w+\mathcal{A} w=L w & \text { in } Q, \\ w(x, t)=0 & \text { on } \Sigma, \\ w(x, T)=w_{T}(x) & \text { in } \Omega,\end{cases}
$$

where $w=\left(w_{j}\right)_{1 \leq j \leq m}$ and $w_{T}=\left(w_{T, j}\right)_{1 \leq j \leq m}$.

Recall $M=\sum_{k=1}^{m} \max _{1 \leq j \leq m}\left\|a_{j k}\right\|_{\infty}^{2}$. Then, using Carleman estimate (3.15) with $f_{j}=0$ $(j=1, \ldots, m)$ and classical tools of controllability (see [18]), we obtain the following observability inequality (with $m$ control forces):

$$
\sum_{j=1}^{m}\left\|w_{j}(0)\right\|_{L^{2}(\Omega)}^{2} \leq C_{T} \sum_{j=1}^{m} \int_{0}^{T} \int_{\omega}\left|w_{j}\right|^{2} d x d t
$$

with $C_{T}=e^{C\left(1+\frac{1}{T}+(1+M) T+M^{\frac{2}{3}}\right)}$ and $C$ a positive constant.

We then obtain the following result. 
Theorem 4.1 The observability inequality (4.5) yields the null controllability result for system (4.3). Namely, for every $y_{0} \in H, \exists v_{j} \in L^{2}(Q)$ such the solution $y$ of (4.3) satisfies

$$
y_{j}(\cdot, T)=0 \quad \text { in } \Omega, \forall j: 1 \leq j \leq m .
$$

4. We give now some results about the Carleman estimate with one observation for a particular coupled parabolic system. Firstly, we consider the case of a $2 \times 2$ coupled parabolic system. It is about obtaining the Carleman estimate with one observation for system (1.1) in the case $m=2$ (noted (1.1) $(m=2)$ ).

Let $k_{j}(j=1,2)$ be $B V$ diffusion coefficients. Recalling that, as in the previous section, $\omega_{0} \Subset \omega \Subset\left(a_{p}, a_{p+1}\right) \Subset \Omega$ and the weight functions

$$
\varphi(x, t)=\frac{e^{\lambda \beta(x)}}{t(T-t)}, \quad \eta(x, t)=\frac{e^{\lambda \bar{\beta}}-e^{\lambda \beta(x)}}{t(T-t)},
$$

where $\beta$ is the function defined through Lemma 2.1.

Let us consider the following assumption.

Assumption 4.1 There exists a constant $b_{0}>0$ such that

$$
a_{21} \geq b_{0} \quad \text { in } \omega \times(0, T) .
$$

Let $\tau \in \mathbb{R}$ and we note

$$
\begin{aligned}
I\left(\tau, k_{l}, y_{l}\right)= & \iint_{Q} e^{-2 s \eta}(s \varphi)^{\tau-1}\left(\left|\partial_{t} y_{l}\right|^{2}+\left|\partial_{x}\left(k_{l} \partial_{x} y_{l}\right)\right|^{2}\right) d x d t \\
& +\lambda^{2} \iint_{Q} e^{-2 s \eta}(s \varphi)^{\tau+1}\left|\partial_{x} y_{l}\right|^{2} d x d t \\
& +\lambda^{4} \iint_{Q} e^{-2 s \eta}(s \varphi)^{\tau+3}\left|y_{l}\right|^{2} d x d t, \quad l=1,2 .
\end{aligned}
$$

Using the results obtained in the previous section and proceeding as in [14], we obtain the following shifted Carleman estimate.

Theorem 4.2 (see [14, Theorem 2.2]) Let j, $k=1$, 2. Let us suppose that $a_{j k} \in L^{\infty}(Q)$. Let $M_{j k}=\left\|a_{j k}\right\|_{\infty}$. We assume that the diffusion coefficients $k_{1}, k_{2} \in B V(\Omega)$ such that $k_{1}, k_{2}$ are of class $\mathcal{C}^{1}$ in $\bar{\omega}$ and satisfy Assumption 1.1. Furthermore, we assume that Assumption 4.1 is satisfied. Then there exist $\lambda_{2}=\lambda_{2}\left(\Omega, \omega,\left\|k_{j}\right\|_{L^{\infty}}\right) \geq 1, s_{2}=s_{2}\left(\lambda_{2}, T, M_{j k}\right)>1$ and a positive constant $C_{2}=C_{2}\left(\Omega, \omega, b_{0}, M_{j k},\left\|k_{j}\right\|_{L^{\infty}}, T\right)$ such that, for any $\lambda \geq \lambda_{2}$ and any $s \geq s_{2}$ and $\epsilon>0$ fixed, the following inequality holds:

$$
\begin{aligned}
& \lambda^{-4+\epsilon} I\left(-3, k_{1}, y_{1}\right)+I\left(0, k_{2}, y_{2}\right) \\
& \leq C_{2}\left[s^{4} \lambda^{4+\epsilon} \int_{0}^{T} \int_{\omega} e^{-2 s \eta} \varphi^{4}\left|y_{2}\right|^{2} d x d t\right. \\
& \left.\quad+s^{-3} \lambda^{-4+\epsilon} \iint_{Q} e^{-2 s \eta} \varphi^{-3}\left|f_{1}\right|^{2} d x d t+\lambda^{2 \epsilon} \iint_{Q} e^{-2 s \eta}\left|f_{2}\right|^{2} d x d t\right]
\end{aligned}
$$

for any solution $\left(y_{1}, y_{2}\right)$ of $(1.1)(m=2)$. 
We also give an observability inequality for a $2 \times 2$ parabolic system by one control force (see [10]). Then we consider system (4.3) with the following changes: $V=\left(0, v_{2}\right)$ and

$$
\mathcal{A}=\left(\begin{array}{cc}
\mathcal{A}_{1} & 0 \\
0 & \mathcal{A}_{2}
\end{array}\right)
$$

By applying Carleman estimate (4.6) with $f_{1}=0, f_{2}=0$, we obtain the following observability inequality (with one control force):

$$
\sum_{j=1}^{m}\left\|w_{j}(0)\right\|_{L^{2}(\Omega)}^{2} \leq e^{C} \int_{0}^{T} \int_{\omega}\left|w_{2}\right|^{2} d x d t
$$

with $C=C(T, M)>0$ and $M=\sum_{k=1}^{2} \max _{1 \leq j \leq 2}\left\|a_{j k}\right\|_{\infty}^{2}$.

We consider now the case of a cascade system, namely the matrix $L$ in system (4.3) has the following structure:

$$
L=\left(\begin{array}{ccccc}
a_{11} & a_{12} & a_{13} & \ldots & a_{1 m} \\
a_{21} & a_{22} & a_{23} & \cdots & a_{2 m} \\
0 & a_{32} & a_{33} & a_{33} \cdots & a_{3 m} \\
\vdots & \vdots & \ddots & \ddots & \vdots \\
0 & 0 & \cdots & a_{m m-1} & a_{m m}
\end{array}\right)
$$

and $V=D v_{1}$, where $D=e_{1}=(1,0, \ldots, 0)^{t}$.

The Carleman estimate obtained in paper [11] can be easily generalized in the case of $B V$ diffusion coefficients by using similar arguments to those in the preceding sections.

Assumption 4.2 There exists a constant $d_{0}>0$ such that

$$
\left|a_{k k-1}\right| \geq d_{0} \quad \text { in } \omega \times(0, T), k=1, \ldots, m .
$$

We note $M_{1}=\max _{2 \leq j \leq m}\left\|a_{k k-1}\right\|_{\infty}$. Then, under Assumption 4.2, we also obtain the following observability inequality by one control force for the cascade system (see [11]):

$$
\sum_{j=1}^{m}\left\|w_{j}(0)\right\|_{L^{2}(\Omega)}^{2} \leq e^{C} \int_{0}^{T} \int_{\omega}\left|w_{1}\right|^{2} d x d t
$$

with $C$ a positive constant, depending on $\Omega, \omega, d_{0}, M_{1}, T$ and $\left\|a_{j k}\right\|_{\infty}$.

\section{Competing interests}

The author declares that he has no competing interests.

\section{Acknowledgements}

The author would like to thank the editor and the anonymous referees for their valuable comments and helpful suggestions, which led to the improvement of the original manuscript.

The author also wishes to thank A Benabdallah, M González-Burgos, J Le Rousseau and N Boussetila for numerous

discussions on the proofs in the paper.

Received: 25 January 2014 Accepted: 29 July 2014 Published online: 25 September 2014 


\section{References}

1. Ladyzhenskaya, O, Solonnikov, V, Uraltseva, N: Linear and Quasilinear Equations of Parabolic Type. Am. Math. Soc, Providence (1968)

2. Doubova, A, Osses, A, Puel, J-P: Exact controllability to trajectories for semilinear heat equations with discontinuous diffusion coefficients. ESAIM Control Optim. Calc. Var. 8, 621-661 (2002)

3. Benabdallah, A, Gaitan, P, Le Rousseau, J: Stability of discontinuous diffusion coefficients and initial conditions in an inverse problem for the heat equation. SIAM J. Control Optim. 46(5), 1849-1881 (2007)

4. Bellassoued, M, Yamamoto, M: Inverse source problem for a transmission problem for a parabolic equation. J. Inverse III-Posed Probl. 14(1), 47-56 (2006)

5. Benabdallah, A, Dermenjian, Y, Le Rousseau, J: Carleman estimates for the one-dimensional heat equation with a discontinuous coefficient and applications to controllability and an inverse problem. J. Math. Anal. Appl. 336, 865-887 (2007)

6. Le Rousseau, J, Robbiano, L: Local and global Carleman estimates for parabolic operators with coefficients with jumps at interfaces. Invent. Math. 183(2), 245-336 (2011)

7. Benabdallah, A, Dermenjian, Y, Le Rousseau, J: Carleman estimates for stratified media. J. Funct. Anal. 260(12), 3645-3677 (2011)

8. Le Rousseau, J: Carleman estimates and controllability results for the one-dimensional heat equation with BV coefficients. J. Differ. Equ. 233, 417-447 (2007)

9. Ammar Khodja, F, Benabdallah, A, Dupaix, C, Kostine, I: Null controllability of some systems of parabolic type by one control force. ESAIM Control Optim. Calc. Var. 11, 426-448 (2005)

10. Ammar Khodja, F, Benabdallah, A, Dupaix, C: Null controllability of some reaction-diffusion with one control force J. Math. Anal. Appl. 320(2), 928-943 (2006)

11. González-Burgos, M, de Teresa, L: Controllability results for cascade systems of $m$ coupled parabolic PDEs by one control force. Port. Math. 67(1), 91-113 (2010)

12. González-Burgos, M, Pérez-García, R: Controllability of some coupled parabolic systems by one control force. C. R. Math. Acad. Sci. Paris 340, 125-130 (2005)

13. Cristofol, M, Gaitan, P, Ramoul, H: Inverse problems for a $2 \times 2$ reaction-diffusion system using a Carleman estimate with one observation. Inverse Probl. 22, 1561-1573 (2006)

14. Cristofol, M, Gaitan, P, Ramoul, H, Yamamoto, M: Identification of two coefficients with data of one component for a nonlinear parabolic system. Appl. Anal. 91(11), 2073-2081 (2012)

15. Benabdallah, A, Cristofol, M, Gaitan, P, Yamamoto, M: Inverse problem for a parabolic system with two components by measurements of one component. Appl. Anal. 88, 683-710 (2008)

16. Benabdallah, A, Cristofol, M, Gaitan, P, de Teresa, L: A new Carleman inequality for parabolic systems with a single observation and applications. C. R. Math. Acad. Sci. Paris 348, 25-29 (2010)

17. Cristofol, M, Gaitan, P, Niinimäki, K, Poisson, O: Inverse problem for a coupled parabolic system with discontinuous conductivities: one-dimensional case. Inverse Probl. Imaging 7(1), 159-182 (2013)

18. Fernández-Cara, E, Guerrero, S: Global Carleman inequalities for parabolic systems and application to controllability. SIAM J. Control Optim. 45, 1395-1446 (2006)

19. Fursikov, A: Optimal Control of Distributed Systems. Translations of Mathematical Monographs, vol. 187. Am. Math. Soc., Providence (2000)

20. Bressan, A: Optimal Hyperbolic Systems of Conservation Laws: The One Dimensional Cauchy Problem. Oxford University Press, Oxford (2000)

\section{Submit your manuscript to a SpringerOpen ${ }^{\circ}$ journal and benefit from:}

- Convenient online submission

- Rigorous peer review

Immediate publication on acceptance

Open access: articles freely available online

- High visibility within the field

- Retaining the copyright to your article 